\title{
Validation of the Intermittent and Constant Osteoarthritis Pain Questionnaire in Patients with Hand Osteoarthritis: Results from the Nor-Hand Study
}

\author{
Marthe Gløersen (1), Pernille Steen Pettersen (i), Tore K. Kvien, and Ida K. Haugen (1)
}

\begin{abstract}
Objective. To examine the validity of a modified Intermittent and Constant Osteoarthritis Pain (ICOAP) questionnaire for assessment of pain in hand osteoarthritis (OA).

Methods. The modified ICOAP-hand questionnaire was administered to 300 patients $[89 \%$ female, median (interquartile range) age: 61 (57-66) yrs] in the Nor-Hand observational cohort study. The questionnaire was completed twice by 31 patients and test-retest reliability was assessed by intraclass correlation coefficients (ICC) for sum scores and weighted $\kappa$ scores for individual items. Internal consistency was assessed by Cronbach's alpha coefficient and item-total correlations. Correlations between the ICOAP-hand questionnaire, the Australian/Canadian Hand OA Index (AUSCAN) hand pain subscale, and pain on a numerical rating scale (NRS) were analyzed using Spearman correlation analyses.

Results. We found a substantial overlap between constant and intermittent pain (46\% reporting constant + intermittent pain and $33 \%$ reporting no pain). Test-retest reliability analysis of ICOAP-hand showed an ICC of 0.89 for the total scale and weighted $\kappa$ values between $0.39-0.70$ for the individual items. Principal component analysis revealed one component with an eigenvalue of 7.9, explaining $72 \%$ of the total variance. Cronbach's alpha coefficient values $>0.93$ and strong item-total correlations proved high internal consistency. ICOAP-hand was strongly correlated with NRS hand pain and the AUSCAN pain subscale.

Conclusion. ICOAP-hand is a reliable pain index that correlates with other available pain questionnaires. However, our results indicate that constant and intermittent pain do not represent separate constructs in hand OA, questioning the usefulness of the 2 subscales. [ClinicalTrials.gov: NCT03083548] (First Release March 15 2019; J Rheumatol 2019;46:645-51; doi:10.3899/jrheum.180835)
\end{abstract}

Key Indexing Terms:

OSTEOARTHRITIS

HAND

PAIN

OUTCOME ASSESSMENT
Hand osteoarthritis (OA) is a prevalent disease, particularly among the elderly, and causes pain and disability ${ }^{1}$. Data collected in the population-based Framingham study showed that $14 \%$ of women and $7 \%$ of men between the ages of 40-84 years have symptomatic hand $\mathrm{OA}^{2}$. In the coming years, both the prevalence and expenses used to treat the disease are expected to grow because of an aging population.

From the Department of Rheumatology, Diakonhjemmet Hospital, Oslo, Norway.

The Nor-Hand study was supported by grants from the South Eastern Norway Regional Health Authority, Jan A. Pahles Foundation, Simon Fougner Hartmanns Family Foundation, and Trygve Gythfeldt's Research Foundation. The funding sources had no involvement in data analyses and reporting of results.

M. Gloersen, Medical Student, Department of Rheumatology,

Diakonhjemmet Hospital; P. Steen Pettersen, MD, Department of Rheumatology, Diakonhjemmet Hospital; T.K. Kvien, MD, PhD, Professor of Rheumatology, Department of Rheumatology, Diakonhjemmet Hospital; I.K. Haugen, MD, PhD, Department of Rheumatology, Diakonhjemmet Hospital.

Address correspondence to M. Glфersen, Department of Rheumatology,

Diakonhjemmet Hospital, Box 23 Vinderen, N-0319 Oslo, Norway.

E-mail: marthe.gl@hotmail.com

Accepted for publication December 3, 2018.
Pain is the main symptom of OA and is a major reason OA patients seek medical help. Hence, OA pain constitutes a great socioeconomic burden ${ }^{3}$. In specialist care, patients with hand OA have similar levels of pain as patients with rheumatoid arthritis (RA) ${ }^{4}$. Pain in hand OA often results in use of simple analgesics and nonsteroidal antiinflammatory drugs, and requires orthopedic surgical interventions in severe cases.

We have limited knowledge about the pain experience in hand OA, partly because of a lack of validated questionnaires. Previous large focus groups arranged in a qualitative multicenter study in 5 European countries identified a range of pain concepts, such as fluctuating pain and psychological consequences of pain, which are not represented in the commonly used instruments to assess hand OA. Hence, the authors recommended that different qualities of pain should be considered for inclusion in pain questionnaires ${ }^{5}$.

The Australian/Canadian Hand OA Index (AUSCAN) is the only currently available validated self-administered questionnaire to measure pain in hand $\mathrm{OA}^{6,7}$, in addition to the numerical rating scale (NRS) or the visual analog scale

Personal non-commercial use only. The Journal of Rheumatology Copyright $($ C 2019. All rights reserved. 
(VAS). However, use of AUSCAN is restricted by copyright, and it measures only pain intensity during different activities. It does not assess how much pain affects patients in daily life, such as how much it interferes with their sleep, mental health, or quality of life. Similarly, the NRS and VAS detect pain intensity only, and do not assess the complex manifestations of pain in hand OA. Consequently, additional validated self-administered questionnaires for assessment of pain in hand $\mathrm{OA}$ are required.

The Intermittent and Constant Osteoarthritis Pain (ICOAP) questionnaire measures both intermittent and constant pain in hip and knee $\mathrm{OA}^{8,9}$. The questionnaire was developed in focus groups. It is customized to detect and differentiate between 2 types of pain acknowledged as important by patients with OA: one dull, persistent and aching type of pain, and one intense and unpredictable type of pain that occurs sporadically ${ }^{10}$. Validity and responsiveness of ICOAP are proven to be good in knee and hip $\mathrm{OA}^{8,11,12,13,14,15}$. However, no previous study has assessed the validity of the ICOAP questionnaire in hand OA, although the questionnaire may be an important contribution to the limited number of previously validated questionnaires to cover more of the concepts recognized as important by patients with hand OA in previous focus groups 5 .

In our current study, we aimed to explore the validity of a modified version of the ICOAP questionnaire (ICOAP-hand), by investigating the test-retest reliability and internal consistency, in addition to correlations with the AUSCAN pain subscale and NRS hand pain.

\section{MATERIALS AND METHODS}

Study participants. The Nor-Hand study is a large hospital-based observational cohort study including 300 patients with hand $\mathrm{OA}^{16}$. Data from the baseline examination (2016-2017) were used in the current study. Based on the available funding, it was found feasible to include 300 patients in the Nor-Hand study. Patients between 40-70 years of age with hand OA by clinical examination or ultrasound were recruited to the study through the rheumatology outpatient clinic at Diakonhjemmet Hospital in Oslo, Norway. Patients were excluded if the following diagnoses were present or suspected: psoriasis, psoriatic arthritis, spondyloarthritis, RA, and hemochromatosis. The detailed inclusion and exclusion criteria are described in the protocol ${ }^{16}$. A flowchart demonstrating the selection of patients is shown in Figure 1.

The study was approved by the Norwegian Regional Committee for Medical and Health Research Ethics (Ref. no: 2014/2057), and all patients received oral and written information and signed the informed consent form. Questionnaires. All patients answered the ICOAP-hand, AUSCAN, and NRS questionnaires in an electronic case report form, or alternatively in paper form, if needed. All questionnaires were administered in Norwegian.

AUSCAN is a disease-specific questionnaire that measures pain based on 5 questions in patients with hand OA. Each of the 5 questions has 5 response options [none, mild, moderate, severe, extreme (scale 0-4)], and the pain sum score ranges from 0 to 20 . The pain questions evaluate pain during the last $48 \mathrm{~h}$ at rest and when the patients are gripping, lifting, turning, and squeezing objects ${ }^{6,7}$. The AUSCAN index has been translated into Norwegian, and is shown to be valid and reliable ${ }^{17,18}$. In the current analyses, we did not use the stiffness (1 question) and difficulties with daily activities (9 questions) domains of AUSCAN.

The NRS question examines pain in the hands during the last $24 \mathrm{~h}$ on a
0-10 scale, where 0 represents no hand pain and 10 represents severe hand pain.

The ICOAP-hand questionnaire includes the same questions as in the original ICOAP questionnaire ${ }^{8}$, except for replacement of "knee/hip" with "hand." It contains 5 questions about constant pain and 6 questions about intermittent pain in the hands during the last week. Both subscales include 5 questions about the following: pain intensity, how much this pain has affected sleep and quality of life, as well as how frustrated/annoyed and how worried/upset the patients have been because of pain during the last week. There are 5 response options for each question $(0=$ not at all, $1=$ mildly, $2=$ moderately, $3=$ severely, $4=$ extremely). In addition, the intermittent pain subscale includes a question about pain frequency, which has 5 response options $(0=$ never, $1=$ rarely, $2=$ sometimes, $3=$ often, $4=$ very often $)$. The sum scores of the subscales are created by summation of the responses to the 5 questions about constant pain ( $0-20$ scale) and the 6 questions about intermittent pain ( $0-24$ scale), respectively. A total ICOAP score is generated by adding the 2 subscales ( $0-44$ scale). The subscale scores for constant pain (0-20), intermittent pain (0-24), and the total score (0-44) of the ICOAP questionnaire are normalized to a scale from 0 (no pain) to 100 (extreme pain) by dividing the sum score with the maximum score and multiplying this by 100 .

To evaluate the test-retest reliability, the questionnaires were readministered in paper form to 31 patients.

Statistical analysis. Mean and median scores were calculated for normally and non-normally distributed data, respectively. A Kruskal-Wallis $\mathrm{H}$ test was performed to investigate whether there was a statistically significant difference in duration of symptoms between the 4 groups of patients with no/mild constant or intermittent pain, intermittent pain only, constant pain only, and both constant and intermittent pain.

Test-retest reliability was evaluated by intraclass correlation coefficients (ICC) for the sum scores and weighted $\kappa$ values for the individual items (0-0.20: poor, 0.21-0.40: fair, 0.41-0.60: moderate, $0.61-0.80$ : good, and $0.81-1.00$ : very good reliability) ${ }^{19,20}$.

Further, internal consistency of ICOAP-hand was assessed by item-total correlations, inter-item correlations, and Cronbach's alpha coefficient. The average of the inter-item correlations is recommended to be between 0.4 and $0.5^{21}$. Cronbach's alpha coefficient values $>0.7$ are considered satisfactory 22 Cronbach's alpha coefficient values $>0.9$ may indicate item redundancy ${ }^{21}$.

Principal component analysis (PCA) was used to assess the scale structure and investigate whether the ICOAP-hand questionnaire represents one single construct, or whether it is favorable to assess constant and intermittent pain separately. Factors were extracted based on the scree plot and the Kaiser criterion, which says that only factors with an eigenvalue of 1 or more should be retained.

External construct validity was assessed by calculation of correlations between the ICOAP-hand questionnaire, the AUSCAN pain subscale, and the NRS question using Spearman correlation coefficients.

Statistical analyses were performed using SPSS statistics version 24 (IBM), and $\mathrm{p}$ values $<0.05$ were considered statistically significant.

\section{RESULTS}

Patient characteristics. The patient characteristics are presented in Table 1. For the ICOAP-hand questionnaire, we found a substantial overlap between constant and intermittent pain. In total, 139 (46.3\%) patients reported both constant and intermittent pain. These patients graded at least 1 question in both subscales of the questionnaire with grade 2 (moderate pain) to grade 4 (extreme pain). No/mild constant pain and no/mild intermittent pain were reported by 99 patients $(33.0 \%)$. These patients graded all questions in both subscales of the questionnaire with grade 0 (no pain) to grade 1 (slight pain). Intermittent pain only and constant pain only

Personal non-commercial use only. The Journal of Rheumatology Copyright $\odot$ (2019. All rights reserved 


\section{$\mathrm{N}=431$ underwent screening}

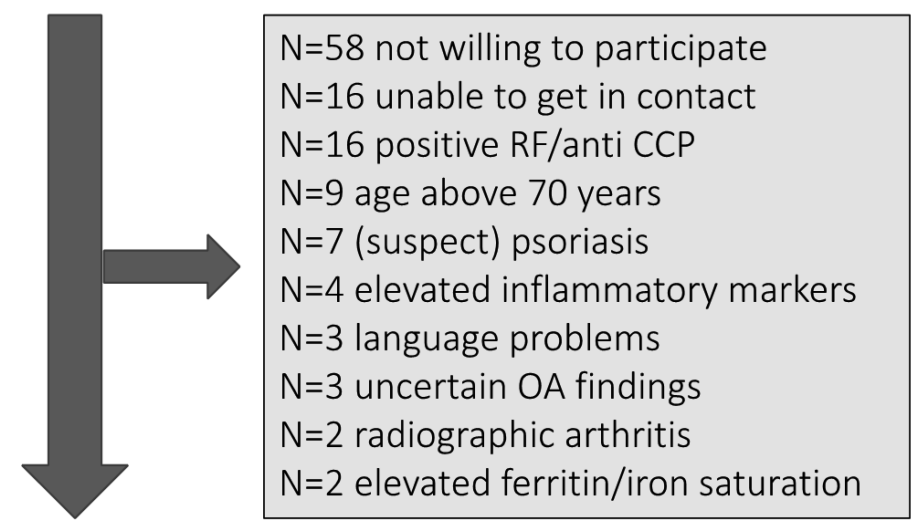

\section{$\mathrm{N}=311$ were examined}



\section{$\mathrm{N}=300$ included in the Nor- Hand cohort}

Figure 1. Flowchart of the Nor-Hand study (2016-2017). RF: rheumatoid factor; anti-CCP: anticyclic citrullinated peptide antibodies; OA: osteoarthritis.

Table 1. Patient characteristics.

\begin{tabular}{lc}
\hline Characteristics & Values \\
\hline Age, yrs & $61.0(56.7-65.9)$ \\
Body mass index, $\mathrm{kg} / \mathrm{m}^{2}$ & $25.5(23.2-29.3)$ \\
Women, $\mathrm{n}(\%)$ & $266(88.7)$ \\
Fulfillment of the ACR criteria for hand OA, $\mathrm{n}(\%)$ & $278(92.7)$ \\
Duration of symptoms, yrs & $6.0(3.0-13.0)$ \\
AUSCAN pain sum score (0-20), mean (SD) & $8.2(4.0)$ \\
NRS hand pain $(0-10)$, mean (SD) & $3.8(2.3)$ \\
ICOAP-hand constant pain sum score (0-100) & $25.0(5.0-40.0)$ \\
ICOAP-hand intermittent pain sum score $(0-100)$ & $25.0(12.5-45.8)$ \\
ICOAP-hand total pain sum score (0-100) & $25.0(11.4-40.9)$ \\
\hline
\end{tabular}

Values are median (IQR) unless otherwise specified. ACR: American College of Rheumatology; OA: osteoarthritis; AUSCAN: Australian/ Canadian Osteoarthritis Hand Index; NRS: numerical rating scale; ICOAP: Intermittent and Constant Osteoarthritis Pain; IQR: interquartile range. were present in $38(12.7 \%)$ and $24(8.0 \%)$ patients, respectively.

Median (interquartile range) duration of symptoms among those with no/mild constant or intermittent pain was 5 (2-11) years, whereas it was 7 (3-17) years among patients with constant pain only, $6(2.5-12)$ years among patients with intermittent pain only, and $6(3-16)$ years among patients with both constant and intermittent pain. There was no statistically significant difference in duration of symptoms between these 4 groups ( $p=0.12$; data not shown).

A small floor effect was identified in the constant pain subscale, because $47(15.7 \%)$ had the lowest score and 0 $(0 \%)$ had the highest score in this subscale. No floor or ceiling effects were seen in the intermittent pain subscale or in the total pain score, because $19(6.3 \%)$ had the lowest score and $0(0 \%)$ had the highest score in the intermittent pain

Personal non-commercial use only. The Journal of Rheumatology Copyright (c) 2019. All rights reserved. 
subscale, and $13(4.3 \%)$ had the lowest score and $0(0 \%)$ had the highest score in the total pain score.

Test-retest reliability. The pain questionnaires were completed twice by 31 patients, and the median (min, max) time interval between the first and second completion was 0 $(0,7)$ days. Test-retest reliability was moderate to good for the ICOAP-hand questionnaire (Table 2), and similar values were shown for AUSCAN pain (ICC 0.65, 95\% CI $0.39-0.82$ ) and NRS pain (ICC $0.78,95 \%$ CI $0.59-0.89$ ).

Internal consistency. The ICOAP-hand questionnaire showed high internal consistency (Cronbach's alpha coefficient 0.96 for the total scale and 0.93 for both subscales), and deletion of items one by one did not increase the Cronbach's alpha coefficient values. Item-total correlations ranged between 0.75 and 0.87 for the constant pain subscale and between 0.73 and 0.87 for the intermittent pain subscale. Inter-item correlations ranged between 0.65 and 0.87 for the constant pain subscale and between 0.57 and 0.87 for the intermittent pain subscale. Missing data in this analysis were $15(5 \%)$.

$P C A$. PCA of the ICOAP-hand questionnaire extracted 1 component with an eigenvalue of 7.9 , explaining $71.6 \%$ of the total variance. Both the scree plot and the Kaiser criterion agreed on extracting 1 single component. The PCA component loadings are listed in Table 2.

External construct validity. The ICOAP-hand total scale and subscales were strongly correlated with the AUSCAN pain subscale and the NRS pain questionnaire (all Spearman $\rho$ correlation coefficients with $\mathrm{p}<0.001$; Table 3 ).

\section{DISCUSSION}

The ICOAP questionnaire was developed for assessment of pain in knee and hip $\mathrm{OA}$ and numerous studies have evaluated its validity in these 2 conditions. Previous focus groups with hand OA patients ${ }^{5}$ identified many concepts that the patients found important, but several of these were not included in the commonly used questionnaires. However, some of these concepts are included in the ICOAP questionnaire, and to our knowledge, the Nor-Hand study is the first to assess the validity of this questionnaire in hand OA. The results from the current study showed that the ICOAP-hand questionnaire is reliable and valid also for assessment of hand OA pain. However, the large overlap between the constant and intermittent pain subscales, which was unrelated to the symptom duration, and the detection of only 1 component in our PCA, question the importance of assessing both these pain aspects in patients with hand OA.

High values of Cronbach's alpha coefficient $(>0.9)$ for both subscales and the total score, as well as large item-total correlations $(>0.7)$ proved that the items of the scale are closely related as a group but may also indicate item redundancy. The identification of possible redundant items in the ICOAP-hand questionnaire (e.g., by Rasch analyses) was outside the scope of the current study. A Rasch analysis of ICOAP has been performed in knee OA, and indicated that the constant and intermittent pain subscales fit the Rasch model if a few items were deleted ${ }^{23}$.

The high values of Cronbach's alpha coefficient corresponded with the PCA, which extracted a single component. These results indicated adequate homogeneity to create 1 summative scale of the ICOAP-hand questionnaire. Hence, using scores from the 2 subscales (constant and intermittent pain) in addition to the total score may not provide additional information. Hawker, et al concluded based on their PCA of ICOAP in hip and knee OA that it was not clear whether a

Table 2. Missing data, principal component analysis (PCA) component loadings, item-total correlations, and test-retest reliability of ICOAP-hand questionnaire.

\begin{tabular}{|c|c|c|c|c|}
\hline Variables & $\begin{array}{c}\text { Missing } \\
\text { Data, n (\%) }\end{array}$ & $\begin{array}{c}\text { PCA } \\
\text { Component } \\
\text { Loadings }\end{array}$ & $\begin{array}{l}\text { Item-total } \\
\text { Correlations }\end{array}$ & $\begin{array}{l}\text { Test-retest } \\
\text { Reliability* }\end{array}$ \\
\hline ICOAP-hand total pain & $0(0)$ & & & $0.89(0.79-0.95)$ \\
\hline ICOAP-hand constant pain & $0(0)$ & & & $0.85(0.72-0.93)$ \\
\hline 1. Pain intensity & $0(0)$ & 0.79 & 0.75 & 0.57 \\
\hline 2. Influence on sleep & $1(0.3)$ & 0.82 & 0.78 & 0.63 \\
\hline 3. Influence on QoL & $3(1.0)$ & 0.88 & 0.85 & 0.39 \\
\hline 4. Frustration & $1(0.3)$ & 0.88 & 0.84 & 0.70 \\
\hline 5. Worries/concerns & $3(1.0)$ & 0.89 & 0.87 & 0.61 \\
\hline ICOAP-hand intermittent pain & $0(0)$ & & & $0.86(0.73-0.93)$ \\
\hline 1. Pain intensity & $2(0.7)$ & 0.77 & 0.73 & 0.56 \\
\hline 2. Frequency & $2(0.7)$ & 0.81 & 0.78 & 0.50 \\
\hline 3. Influence on sleep & $5(1.7)$ & 0.80 & 0.76 & 0.58 \\
\hline 4. Influence on QoL & $1(0.3)$ & 0.88 & 0.85 & 0.56 \\
\hline 5. Frustration & $2(0.7)$ & 0.89 & 0.87 & 0.70 \\
\hline 6. Worries/concerns & $2(0.7)$ & 0.88 & 0.85 & 0.62 \\
\hline
\end{tabular}

* ICC $(95 \%$ CI) for sum scores and weighted $\kappa$ values for individual items. ICOAP: Intermittent and Constant Osteoarthritis Pain; QoL: quality of life; ICC: intraclass correlation coefficient. 
Table 3. Correlations between ICOAP-hand and other pain questionnaires*.

\begin{tabular}{lccccc}
\hline Variables & $\begin{array}{c}\text { ICOAP-hand } \\
\text { Constant Pain }\end{array}$ & $\begin{array}{c}\text { ICOAP-hand } \\
\text { Intermittent } \\
\text { Pain }\end{array}$ & $\begin{array}{c}\text { ICOAP-hand } \\
\text { Total Pain }\end{array}$ & $\begin{array}{c}\text { AUSCAN } \\
\text { Pain }\end{array}$ & $\begin{array}{c}\text { NRS-hand } \\
\text { Pain }\end{array}$ \\
\hline ICOAP-hand constant pain & 1.0 & 0.83 & 0.95 & 0.74 & 0.74 \\
ICOAP-hand intermittent pain & & 1.0 & 0.96 & 0.69 & 0.71 \\
ICOAP-hand total pain & & & 1.0 & 0.75 & 0.76 \\
\hline
\end{tabular}

* All correlations are statistically significant with $\mathrm{p}<0.001$. ICOAP: Intermittent and Constant Osteoarthritis Pain; AUSCAN: Australian/Canadian Osteoarthritis Hand Index; NRS: numerical rating scale.

total score, or intermittent and constant pain subscale scores should be created ${ }^{8}$. However, PCA performed in another study of knee OA showed that intermittent and constant pain items loaded mostly in opposite directions, indicating that intermittent and constant pain should be measured as 2 separate constructs ${ }^{23}$.

The test-retest exercise showed good reliability for the sum scores, whereas the reliability for each item was somewhat lower. The moderate reliability may be due to normal variations in pain intensity, which may fluctuate during the day. We acknowledge that a global question about change in pain intensity between the timepoints for test and retest could have been included, to record this variation in disease activity. Despite a short time interval between the 2 assessments, we expect limited recall bias, because the participants completed the ICOAP-hand questionnaire together with several other questionnaires and questions about demographics and medical history ${ }^{16}$.

Even though the pain indices measure pain during different time intervals ( $24 \mathrm{~h}$ for the NRS, $48 \mathrm{~h}$ for AUSCAN, and 1 week for ICOAP-hand) as well as different aspects of pain, strong correlations were found between the ICOAP-hand, AUSCAN pain, and the NRS, supporting the external validity of the ICOAP-hand questionnaire. One possible advantage of the ICOAP-hand questionnaire is the high correlation with other validated pain outcome measures in hand OA, namely the AUSCAN and NRS. Since AUSCAN is restricted by copyright and therefore has costs related to its use, the ICOAP-hand questionnaire could potentially serve as an alternative. Further, the ICOAP-hand questionnaire includes additional features related to psychological consequences of pain. Focusing on these features of the pain experience may be as important as assessing the pain intensity, especially from a patient perspective ${ }^{5}$.

Moreover, the ICOAP-hand questionnaire differs from the previously validated questionnaire by focusing on both constant and intermittent pain. Previous studies of knee and hip OA have suggested that differentiating these 2 types of pain may be important to gain a better understanding of the pain experience in $\mathrm{OA}^{10}$. However, our results showed a large overlap between the 2 types of pain. The large overlap could be due to long symptom duration in our study. A previous study of knee OA demonstrated progression from intermittent to constant pain interrupted by intermittent unpredictable pain $^{24}$. However, we found no statistically significant difference in symptom duration between patients with no/mild constant or intermittent pain, constant pain only, intermittent pain only, and both constant and intermittent pain.

There are some limitations with this study that need to be considered. First, our data were collected in a hospital-based study, in which all patients were recruited from the outpatient clinic. Hence, the patients included in our study may have higher disease activity than the average patient with hand OA, which could reduce generalizability because most patients with hand $\mathrm{OA}$ are managed in primary care. However, we aimed to include a heterogeneous group of patients with a wide range of severity, pain intensity, and duration of symptoms. Second, we did not investigate the face validity in focus groups in this study. However, a previous qualitative multicenter study in 5 European countries arranged focus groups to identify what patients consider to be the most important aspects of hand $\mathrm{OA}^{5}$. In this study, patients from all 5 countries reported psychological problems, like frustration, anxiety, fear, anger, and sadness as important. The last 2 questions in both subscales of the ICOAP-hand questionnaire cover these psychological aspects. In addition, the ICOAP-hand questionnaire investigates how much hand pain influences sleep, which is reported as important by patients from 3 of the countries in the multicenter study. Patients from 3 countries also reported fluctuating pain as an important aspect of their disease, and this report is compatible with one of the subscales in the ICOAP-hand questionnaire. In total, 4 concepts (psychological problems, influence on sleep, pain in specific body part, and fluctuating pain) identified as important in the focus groups are covered by the ICOAP-hand questionnaire. The AUSCAN subscales cover 6 concepts (pain in specific body part, activity-related pain vs rest or night pain, morning stiffness, difficulty carrying objects, problems with gripping, and handling small gadgets), of which only the first 2 concepts are strictly pain-related. Pain in specific body part is the only common concept. It was emphasized in the focus group study that many concepts reported as important by patients are not covered by the commonly used instruments to assess hand OA. Last, $88.7 \%$ of the participants in the

Personal non-commercial use only. The Journal of Rheumatology Copyright @ 2019 . All rights reserved. 
Nor-Hand study were women. However, we do not believe that the female dominance influences the generalizability of our results, because the high female proportion can probably be explained by the large number of female patients with symptomatic hand OA.

A small floor effect was identified in the constant pain subscale, which may restrict the ability to differentiate the degree of pain among patients with limited symptoms, as well as the ability to detect changes in symptoms among these patients. Two previous studies of knee OA found no floor or ceiling effects present in the ICOAP subscales or in the total score $^{13,23}$.

The ICOAP-hand questionnaire contains the same questions as in the original ICOAP questionnaire developed for assessment of hip and knee OA, except for the replacement of "knee/hip" with "hand." We decided to use "hand" instead of other words such as "fingers," "finger joints," or names of specific finger joints, because naming all those joints would make the questionnaire very extensive and was therefore not practical. Further, "hand" is the terminology used in the AUSCAN questionnaire. A disadvantage of not using a more specific terminology may be that other causes of hand pain (like pain in tendons or carpal tunnel syndrome) can influence the results. In addition, it may be difficult to distinguish between constant and intermittent pain when referring to the whole hand and not individual finger joints. This may be one of the reasons why we found a substantial overlap. OA in different joints could be in different disease stages, and it may be easier for the patients to separate the 2 types of pain if they answer the questionnaire based on their worst finger joint during the last week. However, by using the most painful joint approach we will not be able to investigate the total burden of hand pain. In addition, it may be difficult to report how their mental health is influenced by pain when they cannot take all their painful finger joints into account. Besides, by asking about general hand pain, the ICOAP-hand questionnaire is more similar to other accepted questionnaires to assess hand OA.

Isolated OA in the thumb base may be more similar to OA in weight-bearing joints, and it may be easier for patients to distinguish between constant and intermittent pain in this particular joint. However, the majority of the patients in this study had OA in their interphalangeal joints, either in isolation or in combination with thumb base OA, which is reflected by the fact that $93 \%$ of the patients fulfilled the American College of Rheumatology criteria for hand OA, making us unable to explore this hypothesis.

The ICOAP-hand questionnaire showed high internal consistency and external construct validity, as well as moderate to strong test-retest reliability. The psychological consequences of pain seem relevant in patients with hand OA, but a large overlap was observed between constant and intermittent pain in our study population, questioning the value of the 2 separate subscales. Consequently, the ICOAP questionnaire may not be recommended for use in patients with hand OA. Future studies should explore other pain questionnaires as alternatives to those already available, taking into account the effect of pain on psychological health, emotions, and sleep.

\section{ACKNOWLEDGMENT}

We thank the patients, the project coordinators Karin Magnusson, Elisabeth Mulrooney, and Janicke Magnus, as well as the research assistants and physicians involved in the Nor-Hand study.

\section{REFERENCES}

1. Kloppenburg M, Kwok WY. Hand osteoarthritis - a heterogeneous disorder. Nat Rev Rheumatol 2011;8:22-31.

2. Haugen IK, Englund M, Aliabadi P, Niu J, Clancy M, Kvien TK, et al. Prevalence, incidence and progression of hand osteoarthritis in the general population: the Framingham Osteoarthritis Study. Ann Rheum Dis 2011;70:1581-6.

3. Neogi T. The epidemiology and impact of pain in osteoarthritis. Osteoarthritis Cartilage 2013;21:1145-53.

4. Slatkowsky-Christensen B, Mowinckel P, Loge JH, Kvien TK. Health-related quality of life in women with symptomatic hand osteoarthritis: a comparison with rheumatoid arthritis patients, healthy controls, and normative data. Arthritis Rheum 2007;57:1404-9.

5. Stamm T, van der Giesen F, Thorstensson C, Steen E, Birrell F, Bauernfeind B, et al. Patient perspective of hand osteoarthritis in relation to concepts covered by instruments measuring functioning: a qualitative European multicentre study. Ann Rheum Dis 2009;68:1453-60.

6. Bellamy N, Campbell J, Haraoui B, Buchbinder R, Hobby K, Roth $\mathrm{JH}$, et al. Dimensionality and clinical importance of pain and disability in hand osteoarthritis: Development of the Australian/Canadian (AUSCAN) Osteoarthritis Hand Index. Osteoarthritis Cartilage 2002;10:855-62.

7. Bellamy N, Campbell J, Haraoui B, Gerecz-Simon E, Buchbinder R, Hobby K, et al. Clinimetric properties of the AUSCAN Osteoarthritis Hand Index: an evaluation of reliability, validity and responsiveness. Osteoarthritis Cartilage 2002;10:863-9.

8. Hawker GA, Davis AM, French MR, Cibere J, Jordan JM, March L, et al. Development and preliminary psychometric testing of a new OA pain measure - an OARSI/OMERACT initiative. Osteoarthritis Cartilage 2008;16:409-14.

9. Maillefert JF, Kloppenburg M, Fernandes L, Punzi L, Gunther KP, Martin Mola E, et al. Multi-language translation and cross-cultural adaptation of the OARSI/OMERACT measure of intermittent and constant osteoarthritis pain (ICOAP). Osteoarthritis Cartilage 2009; 17:1293-6.

10. Hawker GA, Stewart L, French MR, Cibere J, Jordan JM, March L, et al. Understanding the pain experience in hip and knee osteoarthritis - an OARSI/OMERACT initiative. Osteoarthritis Cartilage 2008;16:415-22.

11. Davis AM, Lohmander LS, Wong R, Venkataramanan V, Hawker GA. Evaluating the responsiveness of the ICOAP following hip or knee replacement. Osteoarthritis Cartilage 2010;18:1043-5.

12. Mehta SP, Sankar A, Venkataramanan V, Lohmander LS, Katz JN, Hawker GA, et al. Cross-cultural validation of the ICOAP and physical function short forms of the HOOS and KOOS in a multi-country study of patients with hip and knee osteoarthritis. Osteoarthritis Cartilage 2016;24:2077-81

13. Goncalves RS, Meireles AC, Gil JN, Cavalheiro LM, Rosado JO, Cabri J. Responsiveness of intermittent and constant osteoarthritis pain (ICOAP) after physical therapy for knee osteoarthritis. Osteoarthritis Cartilage 2012;20:1116-9. 
14. Risser RC, Hochberg MC, Gaynor PJ, D'Souza DN, Frakes EP. Responsiveness of the Intermittent and Constant Osteoarthritis Pain (ICOAP) scale in a trial of duloxetine for treatment of osteoarthritis knee pain. Osteoarthritis Cartilage 2013;21:691-4.

15. Bond M, Davis A, Lohmander S, Hawker G. Responsiveness of the OARSI-OMERACT osteoarthritis pain and function measures. Osteoarthritis Cartilage 2012;20:541-7.

16. Gloersen M, Mulrooney E, Mathiessen A, Hammer HB, Slatkowsky-Christensen B, Faraj K, et al. A hospital-based observational cohort study exploring pain and biomarkers in patients with hand osteoarthritis in Norway: The Nor-Hand protocol. BMJ Open 2017;7:e016938.

17. Slatkowsky-Christensen B, Kvien TK, Bellamy N. Performance of the Norwegian version of AUSCAN - a disease-specific measure of hand osteoarthritis. Osteoarthritis Cartilage 2005;13:561-7.

18. Moe RH, Garratt A, Slatkowsky-Christensen B, Maheu E, Mowinckel P, Kvien TK, et al. Concurrent evaluation of data quality, reliability and validity of the Australian/Canadian
Osteoarthritis Hand Index and the Functional Index for Hand Osteoarthritis. Rheumatology 2010;49:2327-36.

19. Sim J, Wright CC. The kappa statistic in reliability studies: use, interpretation, and sample size requirements. Phys Ther 2005;85:257-68.

20. Altman DG. Some common problems in medical research. In: Practical statistics for medical research. London: Chapman \& Hall: 1991:396-439.

21. Streiner DL. Starting at the beginning: an introduction to coefficient alpha and internal consistency. J Pers Assess 2003;80:99-103.

22. Bland JM, Altman DG. Cronbach's alpha. BMJ 1997;314:572.

23. Moreton BJ, Wheeler M, Walsh DA, Lincoln NB. Rasch analysis of the intermittent and constant osteoarthritis pain (ICOAP) scale. Osteoarthritis Cartilage 2012;20:1109-15.

24. Neogi T, Niu J, Felson D, Nevitt M, Lewis C, Torner J, et al. Intermittent and constant knee pain patterns: an indicator for radiographic knee OA duration and severity [abstract]? Osteoarthritis Cartilage 2011;19:S135. 